\title{
cFLIP Regulates Death Receptor-mediated Apoptosis in an Ovarian Granulosa Cell Line by Inhibiting Procaspase-8 Cleavage
}

\author{
Fuko MATSUDA ${ }^{1)}$, Naoko INOUE²), Yasufumi GOTO'), Akihisa MAEDA'), Yuan CHENG ${ }^{1)}$, \\ Kazuhiro SAKAMAKI ${ }^{3)}$ and Noboru MANABE ${ }^{1)}$
}

\author{
${ }^{1)}$ Research Unit for Animal Life Sciences, Animal Resource Science Center, Graduate School of Agricultural and Life \\ Sciences, The University of Tokyo, Ibaraki-Kasama 319-0206, ${ }^{2}$ Laboratory of Animal Morphology and Function, Graduate \\ School of Bioagricultural Sciences, Nagoya University, Nagoya 464-8601 and ${ }^{3)}$ Laboratory of Tumor Cell Biology, \\ Department of Life Sciences, Kyoto University, Kyoto 606-8502, Japan
}

\begin{abstract}
More than 99\% of follicles in mammalian ovaries undergo atresia, but the mechanisms regulating the strict selection process are still unclear. Granulosa cell apoptosis is considered the trigger of follicular atresia, which occurs in advance of the death of an oocyte. Cellular FLICE-like inhibitory protein (cFLIP), a homologue of procaspase-8 (also called FLICE), is an intracellular anti-apoptotic protein. It is expressed in granulosa cells of porcine ovaries, where its levels decreases during follicular atresia. We hypothesized that cFLIP regulates granulosa cell apoptosis by acting as a pro-survival factor. In the present study, to further reveal the function of cFLIP in granulosa cells, we examined the anti-apoptotic mechanism of cFLIP using KGN, a human granulosa tumor cell line. Fas-mediated apoptosis was induced by co-treatment with anti-Fas antibody (CH-11), which acts as an agonist of Fas-ligand, and cycloheximide (CHX). When cFLIP was stably expressed in KGN cells following transfection of an expression vector, the Fas-mediated apoptosis was inhibited. Suppression of cFLIP by small interfering RNA (siRNA) spontaneously induced cell death. Silencing of cFLIP promoted cleavage of procaspase-8, and the cell death caused by cFLIP siRNA was completely blocked by a caspase-8 inhibitor (Z-IETD-FMK), indicating that cFLIP regulates apoptosis in KGN cells by inhibiting cleavage of procaspase-8. In conclusion, cFLIP is an essential pro-survival factor for granulosa cells, and it prevents granulosa cell apoptosis by inhibiting procaspase- 8 activation.
\end{abstract}

Key words: Cellular FLICE-like inhibitory protein (cFLIP), Fas-mediated apoptosis, Granulosa cell, KGN cell

(J. Reprod. Dev. 54: 314-320, 2008)

C ellular FLICE-like inhibitory protein (cFLIP: also called CASH, Casper, CLARP, FLAME, I-FLICE, MRIT or usurpin), a homologue of procaspase-8 (also called FLICE), is an endogenous inhibitor of cell death receptor-mediated apoptotic signaling [1-7]. When a cell death ligand binds to its receptor, which contains an intracellular death domain (DD), on the cell surface, the adaptor protein (Fas-associating death domain: FADD), which consists of a DD and a death effector domain (DED), attaches to the intracellular domain of the cell death receptor via homophilic interaction of the DDs. Inactive procaspase-8, which has two DEDs, then binds to the FADD via the homophilic interaction of their DEDs and then procaspase- 8 is dimerized. The dimerized procaspase- 8 is cleaved, and thus active caspase- 8 is formed. As a result, downstream caspases, such as caspase-3, are activated and apoptosis occurs [8-11]. The anti-apoptotic effect of cFLIP comes from its competition with caspase-8. There are two known splice variants of cFLIP, a short (cFLIP $\left.)_{S}\right)$ and long form $\left(\mathrm{cFLIP}_{\mathrm{L}}\right)[5,12]$; cFLIP $_{S}$ consists of two DEDs, and $\mathrm{CFLIP}_{\mathrm{L}}$ contains an additional caspase-like domain. Furthermore, CFLIP $_{\mathrm{L}}$ closely resembles procaspase-8, except that its caspase-like domain lacks proteolytic activity. It is believed that cFLIP binds with FADD and/or procaspase-8 via homophilic interaction of their DEDs, and thus cFLIP inhibits the binding of FADD and procaspase-8. Thus,

Accepted for publication: May 30, 2008

Published online in J-STAGE: July 7, 2008

Correspondence: F. Matsuda (e-mail: Fuko_Minehata@nts.toray.co.jp)
cFLIP disturbs procaspase-8's dimerization and cleavage, resulting in generation of an active caspase- 8 and subsequent apoptotic signal transduction [13, 14].

Recently, the contribution of cFLIP to the regulation of apoptosis in ovarian granulosa cells has been demonstrated using rats and pigs $[12,15,16]$. The strict selection of mammalian ovarian follicles, as more than $99 \%$ of follicles undergo atresia during their growth and development, is precisely regulated by apoptosis of granulosa cells, but many regulatory mechanisms operating at the molecular level remain to be elucidated [17-20]. Since the FasLFas system is one of the best characterized inducers of apoptosis in granulosa cells [21-28] and cFLIP is an inhibitor of Fas-mediated apoptosis in various mammalian cells, it is hypothesized that cFLIP acts as an inhibitor of Fas-mediated apoptosis in granulosa cells and regulates the apoptotic process. However, the detailed role of cFLIP in granulosa cell apoptosis and the molecular mechanism involved in the regulation of apoptosis have not been elucidated.

We evaluated the effect of $\mathrm{CFLIP}_{\mathrm{S}}$ and $\mathrm{CFLIP}_{\mathrm{L}}$ in granulosa cells using the KGN cell line. KGN cells, established from a human granulosa tumor, maintain most of the physiological activities of granulosa cells and are useful for studying the molecular mechanisms regulating physiological phenomena in granulosa cells [29]. We focused on the inhibition of cFLIP in Fas-mediated apoptosis. We examined the effects of the overexpression of cFLIP using KGN cells stably expressing cFLIP and then estimated the suppressive effects of endogenous cFLIP using KGN cells in which cFLIP 
was suppressed by small interfering RNA (siRNA). Cleavage profiles of procaspase- 8 and the effect of caspase inhibitors in KGN cells were also examined.

\section{Materials and Methods}

\section{Cell culture}

KGN cells were cultured in an incubator (Sanyo, Osaka, Japan) in $5 \% \mathrm{CO}_{2}$ in air at $37 \mathrm{C}$. Dulbecco's modified Eagle's medium (DMEM)/F12 (1:1; Invitrogen, Carlsbad, CA, USA) with 10\% (v/ v) fetal bovine serum (FBS; Sigma Aldrich Chemicals, St. Louis, MO, USA), 100 units/ml of penicillin (Sigma) and $100 \mu \mathrm{g} / \mathrm{ml}$ of streptomycin (Sigma) was used for culture of KGN cells.

\section{Induction of Fas-mediated apoptosis}

Fas-mediated apoptosis was induced by addition of $100 \mathrm{ng} / \mathrm{ml}$ of anti-human Fas antibody (CH-11; MBL, Nagoya, Japan) and $5 \mu \mathrm{g} /$ $\mathrm{ml}$ of cycloheximide (CHX; Sigma) to vector-transfected cFLIPexpressing KGN cells or wildtype KGN cells. Cells were plated on 96-well culture plates at $6 \times 10^{3}$ cells/well density (Falcon, Lincoln Park, NJ, USA), and $24 \mathrm{~h}$ after plating, anti-human Fas antibody and/or CHX were added. After 3, 6 or $9 \mathrm{~h}$ of Fas stimulation, cell viability was measured by MTS assay as described below. Caspase activity after $4 \mathrm{~h}$ of Fas stimulation was determined with a CaspACE Assay system (Promega, Madison, WI, USA) as described below.

\section{Cell viability assay}

Cell viability was measured by MTS assay using a CellTiter 96 AQueous One Solution Cell Proliferation Assay kit (Promega) according to the manufacturer's protocol. After addition of CellTiter 96 AQueous One Solution Reagent (20 $\mu \mathrm{l} / 100 \mu \mathrm{l}$ of culture medium) to each well, the plate was incubated for $2 \mathrm{~h}$ at $37 \mathrm{C}$ in a $\mathrm{CO}_{2}$ incubator, and the absorbance at $490 \mathrm{~nm}$ was measured with a 96-well microplate reader (Bio-Rad Laboratories, Hercules, CA, USA).

\section{Caspase activity assay}

Caspase activity was detected with a CaspACE Assay system (Promega), which quantitatively measures caspase-3 protease activity. In brief, KGN cells cultured in a $10-\mathrm{cm}$ cell culture dish (Falcon) were stimulated with anti-human Fas antibody and/or CHX. Four hours after stimulation, the cells were collected by cell scraper and washed with PBS. Cell lysis buffer was then added (10 $\mu \mathrm{l} / 10^{6}$ cells), and the cells were lysed with several cycles of freezethawing. After 15 min on ice, the cell lysate was centrifuged, and the supernatant was used as the cell extract. With $10 \mu \mathrm{l}$ cell extract/ well, reagents were added to 96 -well plates as per the manufacturer's instructions. After incubation at $37 \mathrm{C}$ for $4 \mathrm{~h}$, the absorbance at $405 \mathrm{~nm}$ was measured with a 96 -well microplate reader (Bio-Rad).

\section{Selection of KGN cells stably expressing human cFLIP}

The full-length human $\mathrm{CFLIP}_{\mathrm{S}}$ or $\mathrm{CFLIP}_{\mathrm{L}}$ open reading frame (ORF) was inserted into the vector pCMV-Tag 2B, which contains a neomycin resistance gene (Stratagene, Cedar Creek, TX, USA).
To obtain KGN cells stably expressing cFLIP, pCMV-human cFLIP $_{S}$, pCMV-human cFLIP $_{\mathrm{L}}$ expression vector, or empty vector was transiently introduced into KGN cells using Lipofectamine 2000 (Invitrogen) according to the manufacturer's directions. Briefly, 1 day before the transfection, KGN cells were plated on 24-well culture plates (Falcon). Each plasmid vector (0.8 $\mu \mathrm{g} /$ well) was introduced, and $24 \mathrm{~h}$ later, culture medium containing $400 \mu \mathrm{g} /$ ml of G418 (Sigma) was added to the transfected cells; non-transfected cells were used as a control. The medium was changed every 3 days until all of the cells in the non-transfected control culture were dead. These antibiotic-resistant cells were maintained with a culture medium containing $200 \mu \mathrm{g} / \mathrm{ml}$ of G418.

\section{Inhibition of cFLIP expression by siRNA}

A pSilencer 2.1-U6 neo vector (Ambion, Austin, TX, USA) was used for expressing siRNA according to the manufacturer's instructions. Briefly, the 19-mer siRNA target sequence common to cFLIP $_{\mathrm{S}}$ and $\mathrm{cFLIP}_{\mathrm{L}}$ was AGAGGTAAGCTGTCTGTCG (432-450 of GenBank accession number U97075 and 521-539 of U97074; pSilencer-cFLIP $\mathrm{S}_{\mathrm{S} / \mathrm{L})}$. The target sequence specific to $\mathrm{CFLIP}_{\mathrm{L}}$ was AGAACTATGTGGTGTCAGA (1455-1473 of GenBank accession number U97074; pSilencer-cFLIP ${ }_{\mathrm{L}}$ ). These sequences were designed to make hairpin siRNA and were inserted into the pSilencer vector. As a negative control, a pSilencer 2.1-U6 neo negative control vector (Ambion), which has been confirmed to have no effect on gene silencing in human cells, was used (pSilencer-negative). KGN cells were plated on 96-well cell culture plates and were transiently transfected the next day with $200 \mathrm{ng} /$ well of pSilencer using Lipofectamine 2000 (Invitrogen). At 24 and $48 \mathrm{~h}$ after transfection, cell viability was estimated by MTS assay.

A caspase inhibitor, Z-VAD-FMK (Calbiochem, La Jolla, CA, USA), and caspase-8 inhibitor, Z-IETD-FMK (Calbiochem), were dissolved in dimethyl sulfoxide (DMSO) to a concentration of 50 $\mathrm{mM}$. Subsequently, pSilencer vectors were transiently introduced into KGN cells plated on 96-well plates, and when transfection medium mix was added to the cells, caspase inhibitors were added to the culture medium at a concentration $100 \mu \mathrm{M}$. For the vehicle control, $0.2 \%(\mathrm{v} / \mathrm{v})$ DMSO was added to the control well at the same time. Twenty-four hours after transfection, cell viability was measured by MTS assay.

\section{Western blot analysis}

Cells were washed twice with phosphate-buffered saline (PBS, $\mathrm{pH}$ 7.4), treated with $10 \%(\mathrm{v} / \mathrm{v})$ trichloroacetic acid (TCA; Wako, Osaka, Japan), scraped from the culture plate and lysed in UTD buffer [9 M urea, Wako; 2\% (v/v) Triton-X100, Sigma; 1\% (w/v) $( \pm)$-Dithiothreitol (DTT), Wako]. After treatment with an ultrasonic cell disruptor, $2 \%(\mathrm{w} / \mathrm{v})$ lithium dodecylsulfate (Sigma) and $0.03 \mathrm{M}$ Tris (Sigma) were added. The whole cell lysate was again disrupted with an ultrasonic cell disruptor. The protein concentration was determined with a protein assay dye reagent (Bio-Rad).

Five micrograms of protein/lane was fractionated by sodium dodecyl sulfate-polyacrylamide gel electrophoresis (SDS-PAGE) and transferred to polyvinylidine difluoride (PVDF) membranes (Millipore, Bedford, MA, USA). The membranes were incubated 
in blocking buffer [10 mM Tris-HCl, pH 8.0, $100 \mathrm{mM} \mathrm{NaCl,} \mathrm{0.1 \%}$ (v/v) Tween20, Sigma, and 2.5\% (w/v) bovine serum albumin (BSA), Sigma] for $2 \mathrm{~h}$, followed by mouse anti-human $\mathrm{cFLIP}_{\mathrm{S} / \mathrm{L}}$ antibody (diluted with blocking buffer at 1:300, Santa Cruz Biotechnology, Santa Cruz, CA, USA) or rabbit anti-human caspase-8 antibody (1:200; Santa Cruz Biotechnology) at $4 \mathrm{C}$ for $18 \mathrm{~h}$. After washing in wash buffer [10 mM Tris- $\mathrm{HCl}, \mathrm{pH} 7.5,100 \mathrm{mM} \mathrm{NaCl}$ and $0.1 \%(\mathrm{v} / \mathrm{v})$ Tween20], a secondary antibody [horseradish peroxidase (HRP)-conjugated anti-mouse or anti-rabbit IgG, diluted with washing buffer at 1:2,000; Dako, Glostrup, Denmark] was applied for $1 \mathrm{~h}$ at room temperature. The immune complexes were detected with an ECL Western blot detection reagent hydrogen peroxide kit (Amersham Pharmacia Biotech, Buckinghamshire, UK). The glyceraldehyde-3-phosphate dehydrogenase (GAPDH, used as an intrinsic control) protein level was also examined as an internal control using the same PVDF membrane. Goat anti-human GAPDH antibody (diluted with blocking buffer at 1:250; Santa Cruz Biotechnology) and HRP-conjugated anti-goat IgG (diluted with wash buffer at 1:5,000; Dako) were used for detection of GAPDH.

\section{RNA extraction and reverse transcription-polymerase chain reaction ( $R T-P C R$ )}

Isogen (Wako) was used for extraction of total RNA according to the manufacturer's instructions. First strand cDNA was synthesized from 300 ng of total RNA with a Ready-To-Go T-Primed First-Strand kit (Amersham Pharmacia Biotech). The primers used for PCR were as follows: 5'-ATGTC TGCTG AAGTC ATCCA TCA-3' and 5'-TCAGA TCAGG ACAAT GGGCA-3' for human cFLIP, 5'-TGGGC CGAGG CAAGA TAAG-3' and 5'-GGGGC TTGCT CTTCA TCTTG TA-3' for human CFLIP $_{\mathrm{L}}$ and 5'-TCGTG GAAGG ACTCA TGACC-3' and 5'-ACCTG ACACG TTGGC AGTGG-3' for human GAPDH. The PCR profile was $94 \mathrm{C}$ for 5 min; 30 cycles of $94 \mathrm{C}$ for $30 \mathrm{sec}, 55 \mathrm{C}$ for $30 \mathrm{sec}$ and $72 \mathrm{C}$ for 1 $\mathrm{min}$; and then a final extension period at $72 \mathrm{C}$ for $7 \mathrm{~min}$.

\section{Statistical analysis}

The Student's $t$-test was performed using the StatView IV software (Abacus Concepts, Berkely, CA, USA). Each value represents the mean \pm SEM. Differences of $\mathrm{P}<0.05$ were considered significant.

\section{Results}

\section{Induction of Fas-mediated apoptosis in KGN cells}

We first examined the response of KGN cells to Fas-mediated stimulation. The anti-human Fas antibody $\mathrm{CH}-11$, which is able to induce apoptosis by stimulating Fas-mediated apoptotic signaling, was added to KGN cells with or without $\mathrm{CHX}$. Three, six and nine hours after stimulation, cell viability was measured by MTS assay (Fig. 1A), and at the same time, photographs of the cells were taken (Fig. 1B). When the cells were incubated only with $\mathrm{CH}-11$, significant cell death was not observed (Fig. 1A). Addition of CHX also did not affect the viability of KGN cells (data not shown). However, when $\mathrm{CH}-11$ was added together with $\mathrm{CHX}$, almost all cells
A

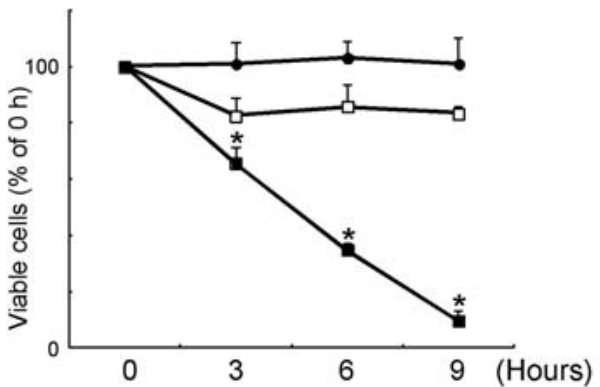

B
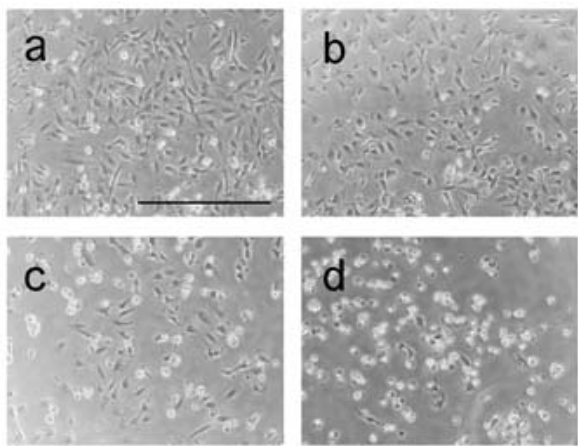

C

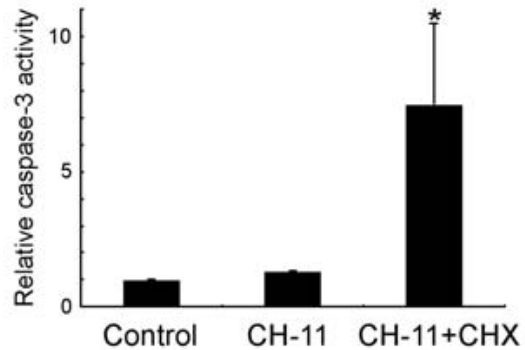

Fig. 1. Anti-human Fas antibody (CH-11) was added to KGN cells with (filled square) or without (empty square) cycloheximide (CHX), and $0,3,6$ and 9 h later, cell viability was examined by MTS assay (A). Filled circles indicate the results for the untreated control. Co-treatment with $\mathrm{CH}-11$ and $\mathrm{CHX}$ killed almost all KGN cells within $9 \mathrm{~h}$. In contrast, addition of just CH-11 did not induce significant cell death. Values represent the means \pm SEM of four independent experiments. *: $\mathrm{P}<0.01$ compared with the untreated control at each time point. Representative photographs of KGN cells before and after addition of $\mathrm{CH}-11$ and $\mathrm{CHX}$ are shown in B (a, b, c and d are the photographs before, $3 \mathrm{~h}$ after, 6 $\mathrm{h}$ after and $9 \mathrm{~h}$ after Fas-stimulation, respectively). The number of detached dead cells increased with time. Scale bar $=500 \mu \mathrm{m}$. Four hours after Fas-stimulation, caspase-3 activity was measured and the relative levels are shown in the graph (C). Addition of $\mathrm{CH}-11$ did not activate caspase-3, but co-treatment with $\mathrm{CH}-11$ and $\mathrm{CHX}$ significantly induced activation of caspase- 3 compared with the untreated control cells. Values represent the means \pm SEM of three independent experiments. *: $\mathrm{P}<0.01$ compared with the control.

died within 9 h (Fig. 1A and B). The cell death induced by Fas was confirmed to be an apoptosis by caspase activity assay, as addition of both $\mathrm{CH}-11$ and $\mathrm{CHX}$ generated caspase-3 activity (Fig. 1C). 
Resistance of KGN cells stably expressing cFLIP to Fasmediated apoptosis

We used KGN cells stably expressing cFLIP or $_{\mathrm{CFLIP}}$ to evaluate the apoptosis-inhibiting ability of cFLIP in granulosa cells. As a negative control, an empty vector containing the neomycin resistance gene was also transfected, and cells selected in the presence of G418 were used. KGN cells expressing cFLIP were confirmed to express more cFLIP compared with wildtype and empty vectortransfected KGN cells (Fig. 2A). Fas-mediated apoptosis was induced in these cells, and cell viability was evaluated after $6 \mathrm{~h}$ (Fig. 2B). While almost all control cells died, the viabilities of both cFLIPs and $\mathrm{CFLIP}_{\mathrm{L}}$-expressing cells were significantly high, approximately 1.8 to 2.0 times the control. Representative photographs of the cells before (Fig. 2C-a, b, and c) and $12 \mathrm{~h}$ after (Fig. 2C-d, e, and f) apoptosis was induced are shown. All control cells (Fig. 2C-a) were dead $12 \mathrm{~h}$ after induction of apoptosis (Fig. 2C-d), but the cFLIPS and CFLIP ${ }_{L}$-expressing KGN cells (Fig. 2C-b and c, respectively) were resistant to Fas-mediated apoptotic signaling (Fig. 2C-e and f, respectively). In particular, $\mathrm{CFLIP}_{\mathrm{L}}$ expressing KGN cells showed strong resistance against Fas-signaling, as there seemed to be no visible difference between before and after Fasstimulation (Fig. 2C-c and f, respectively).

\section{Induction of apoptosis in KGN cells by silencing cFLIP expression}

The siRNA method was used to suppress cFLIP in KGN cells (Fig. 3). Cell viability significantly decreased with suppression of cFLIP (Fig. 3A). In all experiments, no significant difference was found between the $\mathrm{p}$ Silencer-cFLIP $\mathrm{S} / \mathrm{L}$ and $\mathrm{p}$ Silencer-CFLIP $\mathrm{L}_{\mathrm{L}}$ vector-treated cells. Suppression of internal cFLIP mRNA and protein expression by siRNA was confirmed by RT-PCR and Western blot, respectively (Fig. 3B). A positive band was not observed in the Western blot for cFLIP $_{S}$ because the expression of endogenous cFLIPs was trace/negative at the protein level (Fig. 2A).

\section{Apoptosis inhibitory activity of cFLIP caused by the interruption of procaspase- 8 cleavage}

As apoptotic cell death was induced in KGN cells by suppression of cFLIP (Fig. 3), we examined whether or not a caspase-8dependent cascade contributes to apoptosis-inducing mechanisms in granulosa cells. Protein samples were extracted from KGN cells transfected with pSilencer vectors, and procaspase-8 and cleaved caspase-8 (active caspase-8) were detected by Western blot. When cFLIP $_{\mathrm{S} / \mathrm{L}}$ or $\mathrm{CFLIP}_{\mathrm{L}}$ was suppressed, active caspase-8 increased compared with the negative control (Fig. 4). Moreover, we examined the effects of caspase inhibitors on apoptosis in these cFLIPsuppressed KGN cells. KGN cells transfected with pSilencercFLIP vectors underwent apoptotic cell death (Fig. 3A); however, these apoptotic responses were completely blocked by a caspase inhibitor, Z-VAD-FMK (Fig. 5). A caspase-8 inhibitor, Z-IETDFMK, also rescued these KGN cells from apoptosis (Fig. 5).

\section{Discussion}

There are many reports indicating that follicular atresia is dominantly regulated by granulosa cell apoptosis, resulting in the death
A

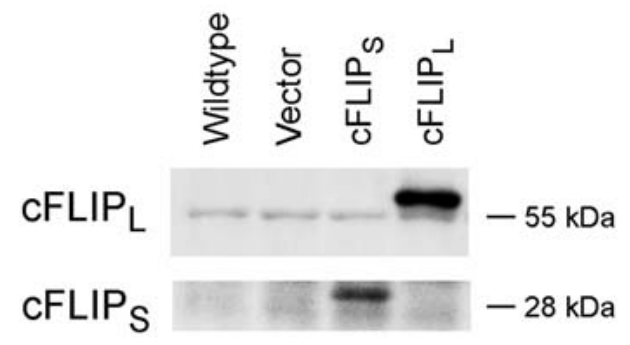

B
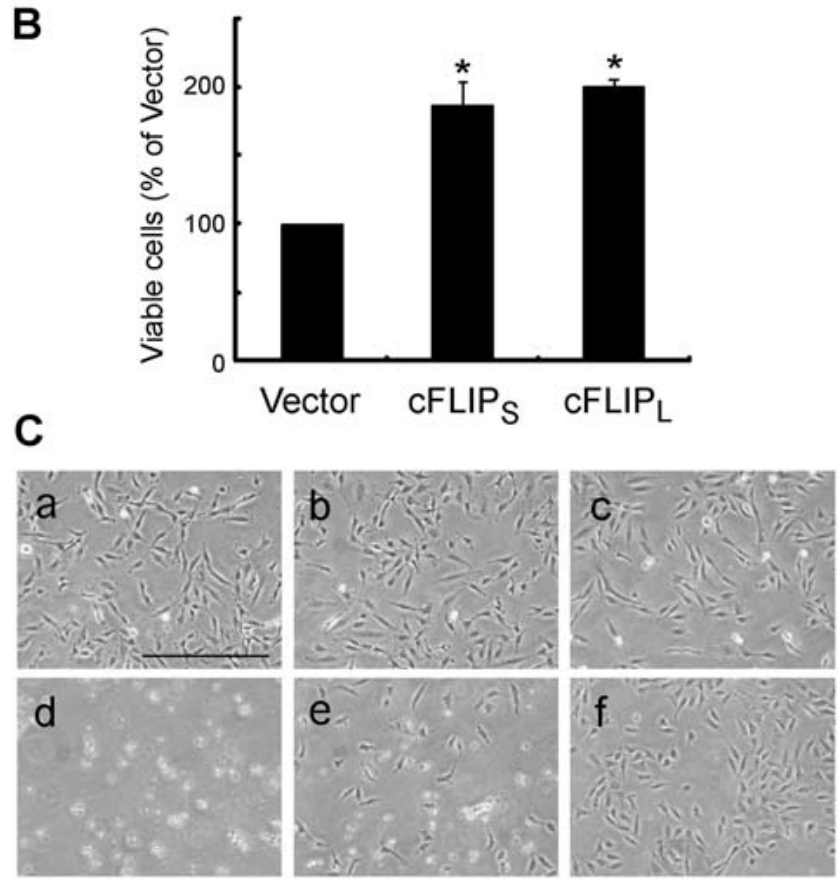

Fig. 2. Human cFLIP $_{\mathrm{S}}$, $\mathrm{cFLIP}_{\mathrm{L}}$, or empty vector (Vector) was transiently introduced into KGN cells and stable cells were obtained by G418 selection. The expression levels of CFLIPS and

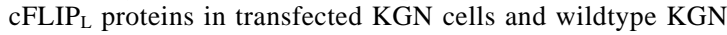
cells (Wildtype) were confirmed by Western blot (A). KGN cells which stably expressing human $\mathrm{CFLIP}_{\mathrm{S}}$ or $\mathrm{CFLIP}_{\mathrm{L}}$ were stimulated by Fas-mediated apoptotic signaling (B and C). Six hours after the anti-human Fas antibody (CH-11) and CHX were added, cell viability was measured by MTS assay (B). Cell viabilities relative to vector control are shown in the graph. The viability of both cFLIP $_{S^{-}}$and cFLIP $_{\mathrm{L}}$-expressing cells was significantly higher than that of the vector control cells. Values represent the means \pm SEM of six independent experiments. *: $\mathrm{P}<0.05$ compared with the vector control. Representative photographs of KGN cells before (C-a, b and c) and $12 \mathrm{~h}$ after (C-d, e and f) addition of $\mathrm{CH}-11$ and $\mathrm{CHX}$ are shown in C. Almost all KGN cells transfected with empty vector (C-a) were detached $12 \mathrm{~h}$ after apoptosis was induced (C-d). KGN cells stably expressing cFLIP $(\mathrm{C}-\mathrm{b})$ or $\mathrm{cFLIP}_{\mathrm{L}}(\mathrm{C}-\mathrm{c})$ were resistant to Fas-mediated apoptosis stimulation (C-e and $\mathrm{f}$, respectively). Scale bar $=500 \mu \mathrm{m}$.

of oocytes [30, 31]. FasL-Fas-mediated signaling is the most characterized death ligand-receptor system involved in granulosa cell apoptosis [21-28]. The binding of FasL and Fas is essential for initiating apoptosis in many types of cells, including granulosa cells; 


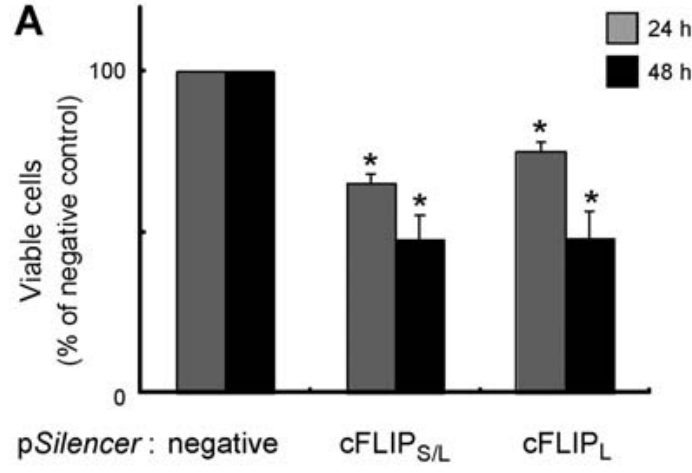

B

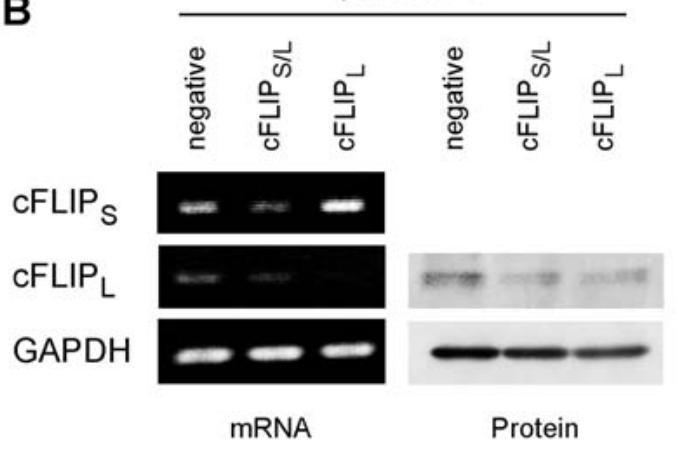

Fig. 3. Silencing of cFLIP by siRNA in KGN cells. The siRNA vectors were introduced into KGN cells and cell viability was examined by MTS assay 24 and 48 h post-transfection (shown in A as gray and black bars, respectively). When cFLIPs and $\mathrm{cFLIP}_{\mathrm{L}}(\mathrm{p}$ Silencer-cFLIP $\mathrm{S} / \mathrm{L}$ ) or only cFLIP $(\mathrm{p}$ Silencer$\mathrm{CFLIP}_{\mathrm{L}}$ ) were suppressed, cell viability was reduced significantly compared with the control (pSilencer-negative; the vector having no effect on gene silencing). Values represent the means \pm SEM of six independent experiments. *: $\mathrm{P}<0.05$ compared with each pSilencer-negative. The downregulation of cFLIP mRNA and protein expression in KGN cells by siRNA was confirmed by RT-PCR and Western blot, respectively (B). Expression of cFLIPs mRNA was downregulated by pSilencer-cFLIP $\mathrm{S}_{\mathrm{S} / \mathrm{L}}$, and $\mathrm{cFLIP}_{\mathrm{L}} \mathrm{mRNA}$ expression was decreased by both pSilencer-cFLIP $\mathrm{S}_{\mathrm{S} / \mathrm{L}}$ and -cFLIP $\mathrm{L}_{\mathrm{L}}$. The protein expression of $\mathrm{CFLIP}_{\mathrm{L}}$ was also suppressed by pSilencer-cFLIP $/ \mathrm{L}$ and -CFLIP $\mathrm{L}_{\mathrm{L}}$. The expression of GAPDH was examined as an internal control.

however, FasL-Fas interaction does not always result in apoptosis. In fact, low levels of FasL and Fas are detected in the granulosa cells of healthy follicles [28, 32]. Furthermore, in cultured cells, stimulation by FasL or agonistic anti-Fas antibody usually cannot induce apoptosis. Co-treatment with FasL or anti-Fas antibody and $\mathrm{CHX}$, an inhibitor of protein synthesis, is necessary for induction of apoptosis in most cultured cells. In primary cultured bovine granulosa cells, FasL alone has no effect on viability [27]. Anti-Fas antibody does not affect the viabilitiy of primary cultured murine granulosa cells, but the cells die when CHX was applied [33]. In the present study, human granulosa tumor cells also underwent apoptosis after addition of anti-Fas antibody and CHX, but did not

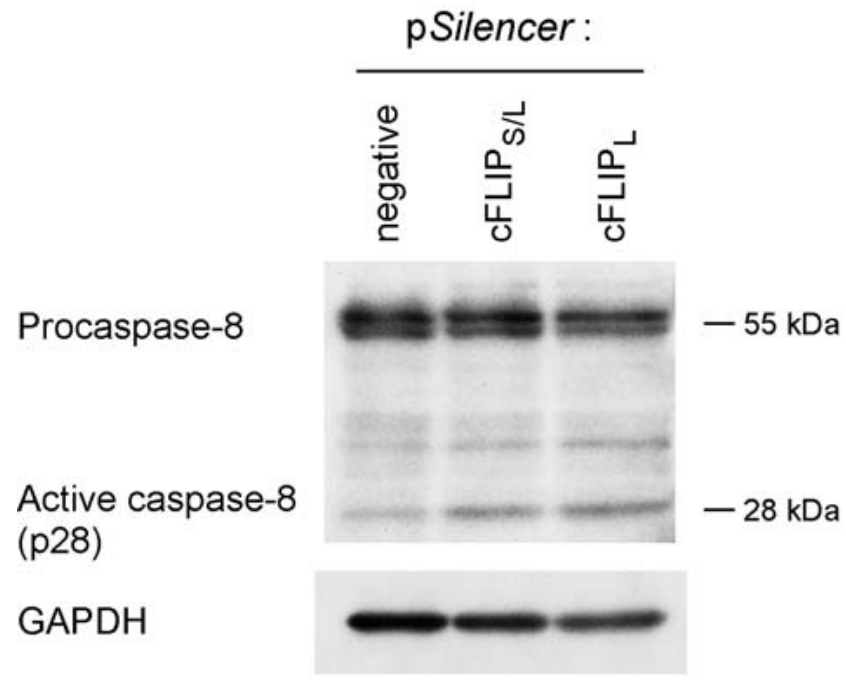

Fig. 4. Cleavage of caspase-8 induced by cFLIP silencing. Proteins were extracted from KGN cells 24 h after pSilencer transfection, and procaspase- 8 and active caspase- 8 were detected by Western blot. Procaspase-8 was detected as a 55$\mathrm{kDa}$ protein, and cleaved active caspase-8 was observed as 28$\mathrm{kDa}$ protein. More activated caspase- 8 was detected in KGN cells transfected with pSilencer-cFLIP ${ }_{\mathrm{S} / \mathrm{L}}$ and $-\mathrm{CFLIP}_{\mathrm{L}}$ than in the pSilencer-negative. The expression of GAPDH was examined as an internal control.

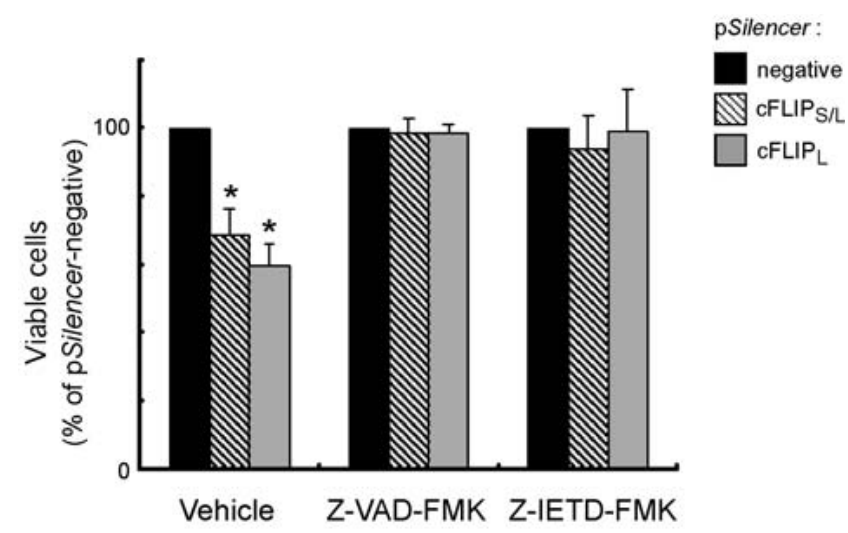

Fig. 5. The effects of caspase inhibitors on apoptosis in cFLIPsuppressed KGN cells. The pSilencer vectors were transfected as in Fig. 3, and $100 \mu \mathrm{M}$ of a caspase inhibitor (Z-VAD-FMK), 100 $\mu \mathrm{M}$ of a caspase-8 inhibitor (Z-IETD-FMK) or only DMSO (Vehicle) was added to the culture medium at the same time the transfection reagent was applied to the cells. Twenty-four hours later, cell viability was measured by MTS assay. Both Z-VADFMK and Z-IETD-FMK resulted in complete blockage of the cell death caused by cFLIP suppression. Values represent the means \pm SEM of four independent experiments. *: $\mathrm{P}<0.05$ compared with pSilencer-negative.

die after addition of anti-Fas antibody alone. Based on these findings, we believe that granulosa cells have an inhibitor protein(s) for the Fas-mediated intracellular pathway. 
It has been reported that $\mathrm{CHX}$ inhibits synthesis of a cell death receptor-mediated apoptotic signaling inhibitor, cFLIP, in some cell lines, which results in induction of apoptosis when co-treated with anti-Fas antibody [34-37]. Recently, we found that cFLIP is expressed in the granulosa cells of porcine ovaries and that its expression is decreased during atresia $[12,16]$. These results suggest that cFLIP may act as a key regulator for Fas-induced granulosa cell apoptosis.

We previously discovered that suppression of porcine cFLIP induces cell death in granulosa cell lines in vitro [38]. However, the mechanisms related to how cFLIP-suppression yields cell death, including downstream caspase's activation, had not yet been elucidated. In the present study, we used a human granulosa tumor cell line, KGN, to further understand the molecular function of cFLIP in granulosa cells. Primary cultured granulosa cells are often used for study of ovarian granulosa cells, but are of limited use when it comes to molecular examinations as spontaneous luteinization occurs shortly after isolation and start of culture. KGN cells express functional FSH receptors, show the same pattern of steroidogenesis as normal granulosa cells and undergo Fasmediated apoptosis due to co-treatment with $\mathrm{CH}-11$ and interferon $\gamma$ in the same manner as normal granulosa cells [29]. Thus, KGN cells retain many important characteristics of granulosa cells. In addition, KGN cells are resistant to gene overexpression and silencing treatments, while primary cultured granulosa cells cannot survive those treatments. For these reasons, we considered them appropriate and useful for study of molecular mechanisms in granulosa cells.

Fas-stimulation resulted in the death of almost all KGN cells within 9 hours. Caspase- 3 was highly activated in KGN cells after Fas-stimulation; this proved that the cell death was apoptosis. We induced overexpression and silencing of cFLIP, and the results showed an anti-apoptotic effect of cFLIP in KGN cells, indicating that sufficient expression of cFLIP inhibits apoptosis and is essential for cell survival in ovarian granulosa cells. KGN cells with stable expression of $\mathrm{CFLIP}_{\mathrm{S}}$ or $\mathrm{CFLIP}_{\mathrm{L}}$ were resistant to Fas-signaling, indicating that both $\mathrm{CFLIP}_{\mathrm{S}}$ and $\mathrm{CFLIP}_{\mathrm{L}}$ act as anti-apoptotic factors in granulosa cells. In the siRNA experiment, there was no significant difference between silencing of both $\mathrm{CFLIP}_{\mathrm{S}}$ and $\mathrm{CFLIP}_{\mathrm{L}}$ (pSilencer-cFLIP $\mathrm{S}_{\mathrm{L}}$ ) and silencing of just $\mathrm{cFLIP}_{\mathrm{L}}(\mathrm{p}$ Silencer$\mathrm{CFLIP}_{\mathrm{L}}$ ). Silencing of $\mathrm{CFLIP}_{\mathrm{L}}$ may be enough to induce apoptosis in human granulosa cells. These results in a human cell line are consistent with our previous findings in porcine ovaries and a porcine granulosa-derived cell line $[16,38]$ indicating that $\mathrm{CFLIP}_{\mathrm{L}}$ in porcine granulosa cells dominantly prevents apoptosis. In rats, $\mathrm{cFLIP}_{\mathrm{S}}$ has an anti-apoptotic effect in cultured granulosa cells [15]. It seems certain that cFLIP acts as an inhibitor of apoptosis in many mammals, but the main isoform may differ among species; that is, cFLIP $_{S}$ acts in incomplete estrus cycle mammals like rodents, while cFLIP $_{\mathrm{L}}$ acts in complete estrus cycle mammals like humans and domestic animals, including pigs.

As mentioned, the KGN cells died when cFLIP was suppressed; however, HeLa cells derived from an epithelial cell tumor were resistant to cFLIP silencing if they were not stimulated by anti-Fas antibody (data not shown), indicating that KGN cells are more sensitive to cFLIP downregulation than HeLa cells. Therefore, cFLIP may be more important for cell survival in granulosa cells than in other cells.

In KGN cells, cleavage/activation of procaspase-8 occurred when cFLIP was downregulated by RNA silencing. Moreover, a caspase-8 inhibitor, Z-IETD-FMK, could prevent the apoptosis caused by cFLIP silencing. Recently, Inoue et al. reported that overexpression of caspase-8 induces apoptosis in granulosa cell lines, including KGN [39]. These facts suggest that apoptosis in granulosa cells is generated by activation of procaspase- 8 and subsequent induction of a caspase cascade, which are prevented by cFLIP.

In the present study, we concluded that cFLIP regulates Fasmediated apoptotic signal transduction in ovarian granulosa cells by inhibiting cleavage of procaspase- 8 , and that it is the essential factor for human granulosa cell survival and maintenance. Considering this report in conjunction with the in vivo findings in our previous papers, cFLIP probably plays crucial roles in determining follicular growth and/or atresia, and this would be a common and fundamental system in the ovaries of many mammalian species. Further studies are needed to reveal the roles of cFLIP in the regulation of granulosa cell survival and death, including the factors that regulate cFLIP expression and/or its function and the molecules that are affected by cFLIP.

\section{Acknowledgements}

We thank Dr. Y Nishi (Kurume University, Kurume, Japan) and Dr. H Nawata (Kyushu University, Fukuoka, Japan) for kindly providing KGN cells. This work was supported by a Grant-in-Aids for Creative Scientific Research (13GS0008), Scientific Research (B 18380164 and S 16108003), Exploratory Research (18658105) to N. M. and by a Research Fellowships for Young Scientists to F. M. from the Japan Society for the Promotion of Science.

\section{References}

1. Goltsev YV, Kovalenko AV, Arnold E, Varfolomeev EE, Brodianskii VM, Wallach D. CASH, a novel caspase homologue with death effector domains. J Biol Chem 1997; 272: 19641-19644

2. Han DK, Chaudhary PM, Wright ME, Friedman C, Trask BJ, Riedel RT, Baskin DG Schwartz SM, Hood L. MRIT, a novel death-effector domain-containing protein, interacts with caspases and BclXL and initiates cell death. Proc Natl Acad Sci USA 1997; 94: 11333-11338.

3. Hu S, Vincenz C, Ni J, Gentz R, Dixit VM. I-FLICE, a novel inhibitor of tumor necrosis factor receptor-1- and CD-95-induced apoptosis. J Biol Chem 1997; 272: 1725517257.

4. Inohara N, Koseki T, Hu Y, Chen S, Nunez G. CLARP, a death effector domain-containing protein interacts with caspase-8 and regulates apoptosis. Proc Natl Acad Sci USA 1997; 94: 10717-10722.

5. Irmler $\mathbf{M}$, Thome $\mathbf{M}$, Hahne $\mathbf{M}$, Schneider $\mathbf{P}$, Hofmann $\mathbf{K}$, Steiner $\mathbf{V}$, Bodmer JL, Schroter M, Burns K, Mattmann C, Rimoldi D, French LE, Tschopp J. Inhibition of death receptor signals by cellular FLIP. Nature 1997; 388: 190-195.

6. Shu HB, Halpin DR, Goeddel DV. Casper is a FADD- and caspase-related inducer of apoptosis. Immunity 1997; 6: 751-763.

7. Srinivasula SM, Ahmad M, Ottilie S, Bullrich F, Banks S, Wang Y, FernandesAlnemri T, Croce CM, Litwack G, Tomaselli KJ, Armstrong RC, Alnemri ES FLAME-1, a novel FADD-like anti-apoptotic molecule that regulates Fas/TNFR1induced apoptosis. J Biol Chem 1997; 272: 18542-18545.

8. Nagata S. Apoptosis by death factor. Cell 1997; 88: 355-365.

9. Ashkenazi A, Dixit VM. Death receptors: signaling and modulation. Science 1998; 281: 1305-1308

10. Wallach D, Varfolomeev EE, Malinin NL, Goltsev YV, Kovalenko AV, Boldin MP 
Tumor necrosis factor receptor and Fas signaling mechanisms. Annu Rev Immunol 1999; 17: 331-367.

11. Hengartner MO. The biochemistry of apoptosis. Nature 2000; 407: 770-776.

12. Goto Y, Matsuda-Minehata F, Inoue N, Matsui T, Maeda A, Manabe N. Porcine (Sus scrofa) cellular FLICE-like inhibitory protein (cFLIP): molecular cloning and comparison with the human and murine cFLIP. J Reprod Dev 2004; 50: 549-555.

13. Krueger A, Baumann S, Krammer PH, Kirchhoff S. FLICE-inhibitory proteins: regulators of death receptor-mediated apoptosis. Mol Cell Biol 2001; 21: 8247-8254.

14. Thome M, Tschopp J. Regulation of lymphocyte proliferation and death by FLIP. Nat Rev Immunol 2001; 1: 50-58.

15. Xiao CW, Asselin E, Tsang BK. Nuclear factor kappaB-mediated induction of Flicelike inhibitory protein prevents tumor necrosis factor alpha-induced apoptosis in rat granulosa cells. Biol Reprod 2002; 67: 436-441.

16. Matsuda-Minehata F, Goto $\mathrm{Y}$, Inoue N, Manabe N. Changes in expression of antiapoptotic protein, cFLIP, in granulosa cells during follicular atresia in porcine ovaries. Mol Reprod Dev 2005; 72: 145-151.

17. Jiang JY, Cheung CK, Wang Y, Tsang BK. Regulation of cell death and cell survival gene expression during ovarian follicular development and atresia. Front Biosci 2003; 8: d222-237.

18. Johnson AL. Intracellular mechanisms regulating cell survival in ovarian follicles. Anim Reprod Sci 2003; 78: 185-201.

19. Manabe N, Goto Y, Matsuda-Minehata F, Inoue N, Maeda A, Sakamaki K, Miyano T. Regulation mechanism of selective atresia in porcine follicles: regulation of granulosa cell apoptosis during atresia. J Reprod Dev 2004; 50: 493-514.

20. Matsuda-Minehata F, Inoue N, Goto Y, Manabe N. The regulation of ovarian granulosa cell death by pro- and anti-apoptotic molecules. J Reprod Dev 2006; 52: 695-705.

21. Quirk SM, Cowan RG, Joshi SG, Henrikson KP. Fas antigen-mediated apoptosis in human granulosa/luteal cells. Biol Reprod 1995; 52: 279-287.

22. Hakuno N, Koji T, Yano T, Kobayashi N, Tsutsumi O, Taketani Y, Nakane PK. Fas/ APO-1/CD95 system as a mediator of granulosa cell apoptosis in ovarian follicle atresia. Endocrinology 1996; 137: 1938-1948.

23. Sakamaki K, Yoshida H, Nishimura Y, Nishikawa S, Manabe N, Yonehara S. Involvement of Fas antigen in ovarian follicular atresia and luteolysis. Mol Reprod Dev 1997; 47: 11-18.

24. Kim JM, Boone DL, Auyeung A, Tsang BK. Granulosa cell apoptosis induced at the penultimate stage of follicular development is associated with increased levels of Fas and Fas ligand in the rat ovary. Biol Reprod 1998; 58: 1170-1176.

25. Peng X, Maruo T, Matsuo H, Takekida S, Deguchi J. Serum deprivation-induced apoptosis in cultured porcine granulosa cells is characterized by increased expression of p53 protein, Fas antigen and Fas ligand and by decreased expression of PCNA. Endocr J 1998; 45: 247-253.
26. Kim JM, Yoon YD, Tsang BK. Involvement of the Fas/Fas ligand system in p53-mediated granulosa cell apoptosis during follicular development and atresia. Endocrinology 1999; 140: 2307-2317.

27. Porter DA, Harman RM, Cowan RG, Quirk SM. Relationship of Fas ligand expression and atresia during bovine follicle development. Reproduction 2001; 121: 561-566.

28. Inoue N, Maeda A, Matsuda-Minehata F, Fukuta K, Manabe N. Expression and localization of Fas ligand and Fas during atresia in porcine ovarian follicles. J Reprod Dev 2006; 52: 723-730.

29. Nishi Y, Yanase T, Mu Y, Oba K, Ichino I, Saito M, Nomura M, Mukasa C, Okabe T, Goto K, Takayanagi R, Kashimura Y, Haji M, Nawata H. Establishment and characterization of a steroidogenic human granulosa-like tumor cell line, KGN, that expresses functional follicle-stimulating hormone receptor. Endocrinology 2001; 142 437-445.

30. Tilly JL. Apoptosis and ovarian function. Rev Reprod 1996; 1: 162-172.

31. Kaipia A, Hsueh AJ. Regulation of ovarian follicle atresia. Аnnu Rev Physiol 1997; 59: 349-363.

32. Kondo H, Maruo T, Peng X, Mochizuki M. Immunological evidence for the expression of the Fas antigen in the infant and adult human ovary during follicular regression and atresia. J Clin Endocrinol Metab 1996; 81: 2702-2710.

33. Quirk SM, Porter DA, Huber SC, Cowan RG. Potentiation of Fas-mediated apoptosi of murine granulosa cells by interferon-gamma, tumor necrosis factor-alpha, and cycloheximide. Endocrinology 1998; 139: 4860-4869.

34. Fulda S, Meyer E, Debatin KM. Metabolic inhibitors sensitize for CD95 (APO-1/Fas)induced apoptosis by down-regulating Fas-associated death domain-like interleukin 1-converting enzyme inhibitory protein expression. Cancer Res 2000; 60: 3947-3956.

35. Kreuz S, Siegmund D, Scheurich P, Wajant H. NF-kappaB inducers upregulate cFLIP, a cycloheximide-sensitive inhibitor of death receptor signaling. Mol Cell Biol 2001; 21: 3964-3973.

36. Mezzanzanica D, Balladore E, Turatti F, Luison E, Alberti P, Bagnoli M, Figini $\mathbf{M}$, Mazzoni A, Raspagliesi F, Oggionni M, Pilotti S, Canevari S. CD95-mediated apoptosis is impaired at receptor level by cellular FLICE-inhibitory protein (long form) in wild-type p53 human ovarian carcinoma. Clin Cancer Res 2004; 10: 5202-5214.

37. Santiago B, Galindo M, Palao G, Pablos JL. Intracellular regulation of Fas-induced apoptosis in human fibroblasts by extracellular factors and cycloheximide. J Immunol 2004; 172: 560-566.

38. Matsuda-Minehata F, Goto Y, Inoue N, Sakamaki K, Chedrese PJ, Manabe N. Antiapoptotic activity of porcine cFLIP in ovarian granulosa cell lines. Mol Reprod Dev 2007; 74: 1165-1170.

39. Inoue N, Matsuda-Minehata F, Goto Y, Sakamaki K, Manabe N. Molecular characteristics of porcine Fas-associated death domain (FADD) and procaspase-8. J Reprod Dev 2007; 53: 427-436 